Applied Physiology, Nutrition, and Metabolism

Canadian Science Publishing Physiologie appliquée, nutrition et métabolisme

\title{
Lactate is Oxidized Outside of the Mitochondrial Matrix in Rodent Brain
}

\begin{tabular}{|r|l|}
\hline Journal: & Applied Physiology, Nutrition, and Metabolism \\
\hline Manuscript ID & apnm-2017-0450.R1 \\
\hline Manuscript Type: & Article \\
\hline Date Submitted by the Author: & 27-Oct-2017 \\
\hline Complete List of Authors: & $\begin{array}{l}\text { Herbst, Eric; University of Guelph, } \\
\text { George, Mitchell; St. Francis Xavier University, Human Kinetics } \\
\text { Brebner, Karen; St. Francis Xavier University, Psychology } \\
\text { Holloway, Graham; University of Guelph, } \\
\text { Kane, Daniel; St. Francis Xavier University, Human Kinetics }\end{array}$ \\
\hline $\begin{array}{r}\text { Is the invited manuscript for } \\
\text { consideration in a Special } \\
\text { Issue? : }\end{array}$ & $\begin{array}{l}\text { Lactate, brain < nervous system, mitochondrial metabolism < metabolism, } \\
\text { respiration, lactate dehydrogenase }\end{array}$ \\
\hline Keywordand & \\
\hline
\end{tabular}


3 Eric A.F. Herbst* ${ }^{1}$, Mitchell A. J. George ${ }^{\dagger 2}$, Karen Brebner ${ }^{\#}$, Graham P. Holloway*, and

4 Daniel A. Kane ${ }^{\dagger}$

5

6 *Department of Human Health and Nutritional Sciences, University of Guelph, Ontario,

$7 \quad$ N1G 2W1, Canada

$8{ }^{\dagger}$ Department of Human Kinetics, and ${ }^{\#}$ Psychology, St. Francis Xavier University,

9 Antigonish, Nova Scotia B2G 2W5, Canada

10

11 Correspondence:

12 Daniel A. Kane

13 Department of Human Kinetics

14 St. Francis Xavier University

151 West Street, Antigonish, NS, Canada B2G 2W5

16 Tel: (902) 867-2251

17 Fax: (902) 867-3904

18 Email: dkane@stfx.ca

19

20

21

22

23

\footnotetext{
${ }^{1}$ Current affiliation: Eric A. F. Herbst; eric.herbst@mq.edu.au; Macquarie University, Sydney, Australia

${ }^{2}$ Current affiliation: Mitchell A. J. George; maigeorge9@gmail.com; University of Calgary, Calgary, AB, Canada
} 


\section{Abstract}

25 The nature and existence of mitochondrial lactate oxidation is debated in the

26 literature. Obscuring the issue are disparate findings in isolated mitochondria, as well as

27 relatively low rates of lactate oxidation observed in permeabilized muscle fibres.

28 However, respiration with lactate has yet to be directly assessed in brain tissue with the

29 mitochondrial reticulum intact. To determine whether lactate is oxidized in the matrix of

30 brain mitochondria, oxygen consumption was measured in saponin-permeabilized mouse

31 brain cortex samples, and rat prefrontal cortex and hippocampus (dorsal) subregions.

32 While respiration in the presence of ADP and malate increased with the addition of

33 lactate, respiration was maximized following the addition of exogenous $\mathrm{NAD}^{+}$,

34 suggesting maximal lactate metabolism involves extra-matrix lactate dehydrogenase.

35 This was further supported when $\mathrm{NAD}^{+}$-dependent lactate oxidation was significantly

36 decreased with the addition of either low-concentration $\alpha$-cyano-4-hydroxycinnamate or

37 UK-5099, inhibitors of mitochondrial pyruvate transport. Mitochondrial respiration was

38 comparable between glutamate, pyruvate, and $\mathrm{NAD}^{+}$-dependent lactate oxidation. Results

39 from the current study demonstrate that permeabilized brain is a feasible model for

40 assessing lactate oxidation, and support the interpretation that lactate oxidation occurs

41 outside the mitochondrial matrix in rodent brain.

42

43 Short title: Mitochondrial lactate oxidation in permeabilized rodent brain.

44

45 Keywords: Lactate, Brain, Mitochondria, Respiration, Lactate dehydrogenase 


\section{Introduction}

48 Lactate was once considered a dead-end waste product of glycolysis produced

49 primarily under anaerobic conditions. However, it is now appreciated that lactate is

50 continuously generated and consumed by oxidative cells (Gladden 2004). Lactate

51 shuttling between tissues serves to distribute oxidizable three-carbon precursors between

52 cells. In the cells of its origin, lactate production can help maintain the free $\mathrm{NAD}^{+}$pool

53 during high rates of glycolysis, and is the major cytosolic redox couple for

$54 \mathrm{NAD}^{+} / \mathrm{NADH}$. Although research examining lactate metabolism has been abundant, the

55 details of its uptake by mitochondria remain an apparent matter of controversy (Passarella

56 et al. 2015, Paventi et al. 2017).

57 In 1985, Brooks proposed a lactate shuttle hypothesis (Brooks 1985) (now

58 referred to as the cell-to-cell lactate shuttle), and later incorporated aspects which

59 suggested that lactate is oxidized by mitochondrial lactate dehydrogenase (LDH) (Brooks

60 et al. 1999a, 1999b). In mammalian skeletal muscle, the notion of direct mitochondrial

61 lactate oxidation has gathered support from some (Brooks et al. 1999b, Dubouchaud et al.

62 2000, Passarella et al. 2008, 2015), and discord from others (Rasmussen et al. 2002,

63 Sahlin et al. 2002, Ponsot et al. 2005, Gladden 2007, Yoshida et al. 2007). Evidence also

64 supports a modified version of the intracellular lactate shuttle (Hashimoto et al. 2006), in

65 which lactate is oxidized to pyruvate prior to its transport across the inner mitochondrial

66 membrane (Szczesna-Kaczmarek et al. 1984, Szczesna-Kaczmarek 1990, Elustondo et al.

67 2013, Jacobs et al. 2013).

68 Previous attempts to resolve the discrepancy in skeletal muscle have been

69 complicated by several factors. First, considering mitochondrial isolation requires 
70 mechanical disruption of the mitochondrial reticulum and differential centrifugation to

71 yield a relatively pure mitochondrial sample, results determined in isolated mitochondria

72 are frequently under scrutiny due to the possibility of cytosolic contamination during the

73 isolation process (Kras et al. 2016). Second, where several studies have reported

74 negligible direct mitochondrial lactate oxidation (Rasmussen et al. 2002, Sahlin et al.

75 2002, Ponsot et al. 2005, Yoshida et al. 2007), work circumventing the need for isolated

76 mitochondria using permeabilized skeletal muscle fibres demonstrated that lactate could

77 be oxidized through the provision of exogenous $\mathrm{NAD}^{+}$(Elustondo et al. 2013, Jacobs et

78 al. 2013). These results identified that $\mathrm{NAD}^{+}$is required to assess mitochondrial

79 respiration supported by lactate, and since $\mathrm{NAD}^{+}$is not membrane permeable, suggest

80 that the LDH enzyme is located outside of the mitochondrial matrix in skeletal muscle.

81 These findings are expected consequences of a marked difference in $\mathrm{NAD}^{+} / \mathrm{NADH}$ redox

82 potential in the cytosol versus mitochondrial matrix (Williamson et al. 1967, Sahlin et al.

83 2002, Jones and Sies 2015).

84 Lactate readily crosses the blood-brain barrier via monocarboxylate transporters

85 (MCTs) (Simpson et al. 2007), and is notably produced and oxidized in the brain

86 (Boumezbeur et al. 2010, Dienel 2012, Schurr 2017). The astrocyte-neuron lactate shuttle

87 hypothesis (Pellerin and Magistretti 2012, Schurr 2017) posits that lactate is exported by

88 glial cells to be taken up and oxidized by neurons as an essential component of neural-

89 glial metabolic cooperation. Isolated mitochondria from the brain offer a potential means

90 of investigating the nature of mitochondrial metabolism in the CNS. However, isolating

91 mitochondria from brain tissue tends to yield a heterogeneous mix of multicellular, if not

92 multiregional mitochondrial populations (Kristian 2010). Isolating brain mitochondria is 
93 further complicated by the extra care necessary to minimize cross-contamination of

94 synaptic versus non-synaptic mitochondria (Kiss et al. 2016).

95 The recent development of the permeabilized brain preparation (Herbst and 96 Holloway 2015) permits direct assessment of lactate oxidation in the brain without 97 disruption of the mitochondrial reticulum. Therefore, the objective of the current study 98 was to investigate and further clarify the nature of mitochondrial lactate oxidation using 99 brain tissue. This was accomplished in both gross mouse cortex samples, as well as 100 specific subregions of rat cortex (prefrontal) and hippocampus (dorsal). We report 101 evidence that maximal mitochondrial lactate oxidation requires extramatrix LDH in 102 rodent brain.

\section{Methods}

105 Animals and reagents

$106 \mathrm{C} 57 \mathrm{BL} / 6$ mice $(n=4)$ were bred on-site at the University of Guelph, and were 107 anesthetized with sodium pentobarbital before cervical dislocation as described 108 previously (Herbst and Holloway 2016). Male Wistar rats $(n=11)$ were purchased from 109 Charles River Laboratories (Montreal), and were anesthetized via an intraperitoneal 110 injection of $250 \mathrm{mg} / \mathrm{ml}$ sodium pentobarbital (Vetoquinol, QC), prior to decapitation and

111 dissection. All other chemicals were purchased from Sigma. All procedures in this study

112 were approved by the University of Guelph Animal Care Committee and the St. Francis

113 Xavier University Animal Care Committee, respectively, and conformed to the standards 114 of the Canadian Council on Animal Care. 
117 Permeabilization of mouse brain tissue for assessment of mitochondrial respiration was

118 performed as described previously (Herbst and Holloway 2015). Briefly, small ( 2 mg)

119 samples were quickly dissected from mouse cortex, weighed, and placed into an

120 Oxygraph-2k (OROBOROS Instruments, Innsbruck, AT) containing a modified MiR05

121 respiration medium (0.5 mM EGTA, $3 \mathrm{mM} \mathrm{MgCl}_{2}, 60 \mathrm{mM} \mathrm{K}$-lactobionate, $10 \mathrm{mM}$

$122 \mathrm{KH}_{2} \mathrm{PO}_{4}, 20 \mathrm{mM}$ HEPES, $110 \mathrm{mM}$ sucrose, $1 \mathrm{~g} / \mathrm{L} \mathrm{BSA}$ ). The method was developed to

123 ensure $\mathrm{H}_{2} \mathrm{O}_{2}$ could be determined, and therefore taurine was removed from the buffer, as

124 originally reported (Herbst and Holloway 2015); taurine was excluded in the current

125 study to be consistent with those experiments. MiR05 media was chosen following

126 preliminary experiments comparing it to another popular, but notably sucrose- and

127 lactobionate-free potassium salt buffer (buffer $z$ (Perry et al. 2013)), due to differences in

128 maximal respiration (Supplementary figure S1). Samples were allowed approximately 5

129 minutes for residual substrates to deplete before saponin or substrate addition to avoid

130 drift during experiments.

131

132 Brain tissue was obtained from euthanized Wistar rats in a manner similar to the mice

133 (Herbst and Holloway 2015), except that brain subregions were dissected using a Brain

134 Matrix (Zivic Instruments, Pittsburgh). Specifically, prefrontal cortex (PFC) and dorsal

135 hippocampus (DH) subregions were dissected. PFC and DH were chosen, because they

136 continue to be areas of intense interest among broad fields of research. Prefrontal cortex

137 plays a major role in executive function, and important functions of the hippocampus

138 include short-to-long-term memory consolidation and spatial memory. Following 
139 dissection, PFC and DH samples were quickly weighed, and placed into the oxygraph.

140 Samples were allowed to incubate for $5 \mathrm{~min}$ prior to permeabilization with saponin (50

$\left.141 \mu \mathrm{g} \cdot \mathrm{mL}^{-1}\right)$. Respirometry was conducted at $37^{\circ} \mathrm{C}$ in $2 \mathrm{~mL}$ MiR05 with constant stirring at

$142750 \mathrm{rpm}$. Instrumental background $\mathrm{O}_{2}$ consumption was corrected using equations

143 determined under the same parameters used for experimental data collection.

145 Titration protocols

146 To ensure that saturating concentrations of $\mathrm{NAD}^{+}$and lactate were used in the subsequent

147 respiratory protocols, titrations were performed on mouse cortical samples with $\mathrm{NAD}^{+}$

$148(0.25,0.5,1,1.5,2.0,2.5,5 \mathrm{mM}$; in the presence of $100 \mathrm{mM}$ lactate $)$ and lactate $(5,10$,

$14920,40,60,80,100 \mathrm{mM}$; in the presence of $2 \mathrm{mM} \mathrm{NAD}^{+}$). To test the individual and

150 summative effects of lactate and $\mathrm{NAD}^{+}$on mitochondrial respiration in the mouse cortex,

$1515 \mathrm{mM} \mathrm{NAD}^{+}$and $80 \mathrm{mM}$ lactate were added separately to the respirometer, followed by

152 their combined addition. Due to previous concerns that lactobionate contained within

153 MiR05 respiration medium may have an inhibitory effect on lactate-mediated respiration

154 (Passarella et al. 2015), we replaced MiR05 with potassium salt buffer $Z$ in the presence

155 of $5 \mathrm{mM} \mathrm{ADP}+4 \mathrm{mM}$ malate $+80 \mathrm{mM}$ lactate $+5 \mathrm{mM} \mathrm{NAD}^{+}$, and compared the two

156 media (Supplementary figure S1).

157 Maximal mitochondrial respiration in mouse cortex was stimulated in the

158 presence of either $10 \mathrm{mM}$ pyruvate, $10 \mathrm{mM}$ glutamate, or $80 \mathrm{mM}$ lactate $+5 \mathrm{mM} \mathrm{NAD}^{+}$.

159 The monocarboxylate transporter inhibitor, $\alpha$-cyano-4-hydroxycinnamate (CHC), was

160 titrated in the presence of glutamate, pyruvate, or lactate $+\mathrm{NAD}^{+}$at $5 \mu \mathrm{M}, 25 \mu \mathrm{M}$ and 50

$161 \mu \mathrm{M}$. CHC and UK-5099 (see subsequent paragraph) are recognized inhibitors of both 
162 monocarboxylate transport in the plasma membrane, but more potently, mitochondrial

163 pyruvate transport (Halestrap and Denton 1974, Halestrap 1975, Poole and Halestrap

164 1993, Gray et al. 2014). Saponin-permeabilization of the plasma membrane removes the

165 role of plasma membrane monocarboxylate transporter inhibition in the current study.

166 Due to slightly different maximal rates of respiration, inhibition experiments were

167 expressed as a percentage of state III respiration for that substrate (control). In separate 168 permeabilized mouse cortex samples, the LDH inhibitor oxamate (Oxm) was also titrated

$169(10,20$ and $40 \mathrm{mM})$ in the presence of $5 \mathrm{mM} \mathrm{ADP}+4 \mathrm{mM}$ malate $+2 \mathrm{mM} \mathrm{NAD}^{+}$and

170 either $80 \mathrm{mM}$ lactate or $10 \mathrm{mM}$ pyruvate $(n=3-4)$.

171

172 Experimental protocol

173 In rat $\mathrm{PFC}$ and $\mathrm{DH}$, the respirometric protocol involved sequential addition of 174 substrates and inhibitors as follows: $5 \mathrm{~mm}$ ADP, $4 \mathrm{~mm}$ malate, $40 \mathrm{~mm}$ lactate, 4 $175 \mathrm{mM} \mathrm{NAD}^{+}, 10 \mu \mathrm{M}$ UK-5099, an inhibitor of mitochondrial pyruvate transport, 10

$176 \mathrm{mM}$ glutamate, and $1 \mathrm{mM}$ aminooxyacetate, an inhibitor of the malate-aspartate shuttle

177 aminotransferases. Malate concentration was chosen based upon separate experiments

178 comparing $4 \mathrm{mM}$ to each $2 \mathrm{mM}$ and $0.5 \mathrm{mM}$ malate (Supplementary figure $\mathrm{S} 2$ ). $J_{2} \mathrm{O}_{2}$ was

179 expressed as picomol $\mathrm{O}_{2} \times \mathrm{s}^{-1} \times \mathrm{mg}^{-1}$ tissue wet weight. ADP was added first to ensure 180 further substrate-stimulated respiration was not dependent on the presence of ADP.

181 Malate was subsequently added to ensure high rates of lactate (and NAD ${ }^{+}$)-stimulated

182 respiration. UK-5099 was added to determine if the rate of lactate-stimulated respiration

183 would be reduced when mitochondrial pyruvate transport was inhibited. Glutamate was

184 added at the end of the protocol to determine if off-target effects of pharmacological 
185 experiments were present. Finally, aminoxyacetate was added as an inhibitor of

186 aminotransferase to determine the potential impact of the malate-aspartate shuttle.

188 Statistical Analysis

189 Statistical analyses were performed using GraphPad Prism 7 (GraphPad Software, Inc.,

190 La Jolla, CA). One-way and two-way ANOVA were used to detect differences in

191 substrate-linked and inhibitor-affected respiration in mouse samples. Two-way ANOVA

192 with repeated measures was used to compare rat PFC to $\mathrm{DH}$ respiration. Bonferroni post

$193 h o c$ tests were used to identify specific differences among dependent variables. Bartlett's

194 test did not support homogeneity of variances. Therefore, all data were square root -

195 transformed prior to analysis. The alpha-level for statistical significance was set at $p=$

196 0.05. All data are reported as means \pm standard deviation.

$198 \quad$ Results

199 Lactate oxidation in permeabilized mouse brain

200 Use of the permeabilized brain preparation provides the opportunity to analyze

201 mitochondrial respiration in the brain without disrupting the mitochondrial reticulum.

202 However, because lactate-stimulated respiration has not been previously assessed in the 203 permeabilized rodent brain, we first established the appropriate concentrations of key 204 substrates required for this preparation. Titration of $\mathrm{NAD}^{+}(0-2.5 \mathrm{mM}$; apparent $\mathrm{Km}$ for

$\left.205 \mathrm{NAD}^{+}: 1.3 \pm 0.5 \mathrm{mM}\right)$ and lactate $(0-100 \mathrm{mM}$; apparent $\mathrm{Km}$ for lactate: $11.2 \pm 3.5 \mathrm{mM})$

206 were performed in separate experiments to ensure that saturating concentrations of the

207 respective substrates were added in subsequent protocols (Fig. 1). Further, where it has 
208 been previously hypothesized that $60 \mathrm{mM}$ lactobionate used in the respiration buffer may

209 inhibit LDH (Passarella et al. 2015), we replaced our buffer with the frequently employed

210 buffer Z (Perry et al. 2013) and observed no increase in respiration (Supplementary figure

211 S1), demonstrating that lactobionate does not have an inhibitory effect on lactate

212 metabolism.

213

214 NAD-dependency on maximal lactate-supported respiration in mouse brain

215 Previous work in skeletal muscle has shown that the provision of exogenous $\mathrm{NAD}^{+}$is

216 required to observe lactate respiration in permeabilized muscle fibers (Elustondo et al.

217 2013, Jacobs et al. 2013). Considering the mitochondrial inner membrane is impermeable

218 to $\mathrm{NAD}^{+}$and NADH (Di Lisa and Ziegler 2001), these previous findings suggest that

219 lactate is oxidized to pyruvate outside of the mitochondrial matrix before transportation

220 across the membrane. To determine whether these findings are corroborated in mouse

221 brain mitochondria, lactate- and $\mathrm{NAD}^{+}$-stimulated respiration were analyzed

222 independently, and in conjunction with one another. While no significant increase in

223 respiration was observed with either lactate or $\mathrm{NAD}^{+}$alone, their combined addition

224 resulted in an approximately $90 \%$ increase in respiration $(P<0.001$; Fig. 2A). Therefore,

225 the requirement for the addition of $\mathrm{NAD}^{+}$not only allays concerns about residual $\mathrm{NAD}^{+}$

226 contamination in the permeabilized brain preparation, but also supports that lactate was

227 oxidized outside of the mitochondrial matrix in these experiments. Moreover, lactate

228 respiration in the presence of $\mathrm{NAD}^{+}$was comparable to complex I-linked respiration

229 stimulated by either pyruvate or glutamate (Fig. 2B). 
231 Respiration supported by NAD-dependent lactate oxidation requires $L D H$ and

232 mitochondrial monocarboxylate transport in permeabilized mouse brain

233 To investigate whether inhibition of $\mathrm{LDH}$ affected $\mathrm{NAD}^{+}$-dependent lactate oxidation,

234 oxamate (inhibitor of $\mathrm{LDH})$ was titrated $(10,20$ and $40 \mathrm{mM})$ in the presence of $5 \mathrm{mM}$

$235 \mathrm{ADP}+4 \mathrm{mM}$ malate, $2 \mathrm{mM} \mathrm{NAD}{ }^{+}$and either $10 \mathrm{mM}$ pyruvate or $80 \mathrm{mM}$ lactate.

236 Whereas the effect of oxamate on pyruvate respiration was negligible, oxamate

237 significantly inhibited $\mathrm{NAD}^{+}$-dependent lactate respiration relative to control (Fig. 3A).

238 However, given reports that oxamate crosses the inner mitochondrial membrane

239 (Halestrap 1975), as well as the possibility that oxamate can affect pyruvate transport into

240 mitochondria (Martin-Requero et al. 1986), it was important to employ inhibitors of other

241 potential aspects of mitochondrial lactate metabolism. To determine whether lactate

242 either enters the mitochondrial matrix directly, or is first converted to pyruvate before

243 transport and oxidation, the mitochondrial pyruvate transport inhibitor $\alpha$-cyano-4-

244 hydroxycinnamate (CHC; (Halestrap 1975)) was used in permeabilized mouse cortex

245 samples. In these experiments, titration of $\mathrm{CHC}$ produced a $>50 \%$ inhibition of both

246 lactate- and pyruvate-stimulated respiration $(P<0.001)$. Together with our observations

247 of inhibition with oxamate, these experiments support that lactate is oxidized to pyruvate

248 prior to being transported across the inner mitochondrial membrane.

249 Because potential off-target effects of pharmacological experiments are always of

250 concern, glutamate respiration was also assessed in the presence of $\mathrm{CHC}$ to serve as a

251 positive control, as glutamate does not interact with mitochondrial monocarboxylate

252 transport and is also a complex I-linked substrate. Glutamate-stimulated respiration was 
253 unaffected by $\mathrm{CHC}$ (Fig. 3B), suggesting that $\mathrm{CHC}$ is specific to monocarboxylate

254 transport and does not negatively impact respiration.

255

256 Respiration supported by NAD-dependent lactate oxidation requires mitochondrial 257 pyruvate transport in permeabilized subregions of rat brain

258 To further explore these findings, we utilized another inhibitor of mitochondrial pyruvate 259 transport in permeabilize rat brain samples. In both rat PFC and DH, a more specific 260 inhibitor of mitochondrial pyruvate transport, UK-5099 (Halestrap 1975, Gray et al. 261 2014) abolished $(P<0.001) \mathrm{NAD}^{+}$-dependent, lactate-supported mitochondrial

262 respiration (Fig. 4). This further emphasizes the necessity of pyruvate transport into the 263 matrix to support lactate oxidation. As with CHC (Fig. 3B), glutamate-stimulated 264 respiration was unaffected by or UK-5099 (Fig. 4), supporting that UK-5099 is also 265 specific to pyruvate transport, and does not negatively impact respiration by affecting the 266 tricarboxylic acid cycle or electron transport chain in these samples. Glutamate 267 respiration was significantly decreased however, following addition of the 268 aminotransferase inhibitor aminooxyacetate (Fig. 4). This suggests that the malate269 aspartate shuttle, which depends on aspartate aminotransferase, is required for 270 glutamate/malate-dependent respiration. While the possibility of off-target effects with 271 any inhibitor is acknowledged, these data suggest that the malate-aspartate shuttle, which 272 depends on aspartate aminotransferase, is required. 


\section{Discussion}

277 The present study sought to examine the functional location of mitochondrial

$278 \mathrm{LDH}$ in rodent brain. We observed an $\mathrm{NAD}^{+}$-dependency of lactate respiration in the

279 cortex and hippocampus, as well as ablated lactate oxidation upon inhibition of

280 mitochondrial pyruvate transport. Taken together, these findings corroborate previous

281 work in muscle suggesting that mitochondrial LDH is located outside of the

282 mitochondrial matrix. However, unlike skeletal muscle, we also observe modest lactate-

283 supported mitochondrial respiration prior to addition of exogenous $\mathrm{NAD}^{+}$. A similar

284 phenomenon is observed in liver, where $\mathrm{NAD}^{+}$remains bound to $\mathrm{LDH}$ localized to the

285 intermembrane space, even during acrylamide gel electrophoresis (Skilleter and Kun

286 1972). Thus, residual binding of $\mathrm{NAD}^{+}$to $\mathrm{LDH}$ outside of the matrix in our preparation is

287 plausible.

288 The nature of mitochondrial lactate metabolism has been a contentious topic. This

289 may be due, at least in part, to a heavy reliance on some indirect techniques (e.g.,

290 spectrophotometric monitoring of NADH change), and generalizations among species,

291 tissues and cell types. Recently, the mitochondrial metabolism of lactate in cultured

292 cancer cell lines was investigated more directly, using ${ }^{13} \mathrm{C}$-labeled lactate and high-

293 resolution mass spectrometry (Chen et al. 2016). The results support the notion of a

294 mitochondrial L-lactate carrier (Taylor 2017) and matrix LDHB in lactate-supported

295 lipogenesis in proliferating, immortalized cells (Chen et al. 2016). Whether this

296 observation translates to mitochondria in mature, differentiated tissues like skeletal

297 muscle and brain has not, to our knowledge, been reported. 
Questions about the purity of isolated mitochondria and a mismatch in pyruvate-

299 lactate respiration in oxidative muscle fibers have also not been entirely explained. In the

300 brain, however, analysis of respiration in permeabilized tissue demonstrates similar

301 observable oxidation rates between both lactate and pyruvate, which suggests the brain

302 may provide a better model of studying mitochondrial-associated LDH. While lactate is

303 well-established to be produced and oxidized in non-oxidative and oxidative skeletal

304 muscle fibers, respectively, evidence supports the shuttling of lactate in the brain between

305 glia and neurons for similar purposes. As such, LDH isoforms associated with skeletal

306 muscle (LDH-5) and the heart (LDH-1) have also been identified in brain tissue (Bittar et

307 al. 1996, Hashimoto et al. 2008); it is of interest that these isoforms have been found to

308 be expressed in a cell- and region-specific distribution, suggesting different regions of the

309 brain may possess unique requirements for lactate oxidation. Moreover, within any given

310 region, the location of mitochondrial $\mathrm{LDH}$ is of particular importance in understanding

311 the cell-to-cell metabolic cooperation within the brain. The astrocyte-neuron lactate

312 shuttle hypothesis supports that glucose is taken from the circulation and converted to

313 lactate for oxidation in neurons (Bouzier-Sore et al. 2003, Pellerin and Magistretti 2012).

314 An alternative hypothesis posits that the resulting lactate transport to neurons results only

315 in the donation of electrons through the reduction of $\mathrm{NAD}^{+}$prior to the shuttling of

316 pyruvate back to astrocytes for oxidation (reviewed in (Dienel 2012)). While the present

317 study does not expand on these hypotheses, the commitment of lactate to the

318 mitochondrial matrix would prevent the theoretical recycling of pyruvate, where evidence

319 presented here and elsewhere (Cerdan et al. 2006) support that neither lactate nor

320 pyruvate are directly or necessarily committed to mitochondrial oxidation. 
322 skeletal muscle (Elustondo et al. 2013, Jacobs et al. 2013), as well as here in the brain,

323 provide evidence that mitochondrial LDH is not located exclusively, if at all, in the

324 mitochondrial matrix of normal, differentiated brain tissue. Though previous studies have 325 demonstrated that the majority of cellular $\operatorname{NAD}^{+}(>75 \%)$ is located inside the 326 mitochondria (Tischler et al. 1977, Di Lisa and Ziegler 2001), the cytosolic

$327 \mathrm{NAD}^{+} / \mathrm{NADH}$ redox potential is reported to greatly exceed the mitochondrial

$328 \mathrm{NAD}^{+} / \mathrm{NADH}$ potential ((Williamson et al. 1967, Jones and Sies 2015); see (Sahlin et al.

329 2002) for discussion)), suggesting that lactate oxidation is favored outside of the

330 mitochondrial matrix. In the cytosol, this is due to the equilibrium constant for the

331 associated redox couples (namely, lactate/pyruvate) combined with influential protein

332 binding sites for NADH (Jones and Sies 2015); changes in NADH are therefore primarily

333 mitochondrial (Sun et al. 2012, Blacker and Duchen 2016). Indeed, consistent with the

334 present study, lactate oxidation only occurs when $\mathrm{NAD}^{+}$is added. These results are

335 consistent with ideas about the nature of mitochondrial lactate oxidation in which LDH

336 acts external to the matrix (Gladden 2007, 2008, Hashimoto et al. 2008, Kane 2014,

337 Rogatzki et al. 2015).

338 Although little is known about the nature of $\mathrm{NAD}^{+}$synthesis and transport into

339 the mitochondria, current evidence supports that the concentration of $\mathrm{NAD}^{+}$within 340 different compartments is highly regulated (di Lisa 2001b). As such, where NAD ${ }^{+}$

341 concentrations have been shown to respond to changes in ATP availability in the cytosol,

342 this is not true in the matrix (Vinogradov et al. 1972, Devin et al. 1997, Di Lisa and 343 Ziegler 2001). Therefore, extramatrix LDH may help couple adaptations in $\mathrm{NAD}^{+}$ 
344 concentrations to changes in the availability of lactate as an energy substrate. This would

345 be an important adaptive mechanism in the brain in particular, where substrate selectivity

346 is regulated uniquely between cell types and brain regions. Though the relationship

347 between cytosolic $\mathrm{NAD}^{+}$concentrations and lactate availability has yet to be investigated,

348 the present data supports the $\mathrm{NAD}^{+}$-dependent nature of lactate oxidation in the cytosol

349 of rodent brain.

350 The centrality of lactate to neuroenergetics (Schurr 2017), and to metabolic

351 physiology in general (Hui et al. 2017), is undeniable. The experimental freedoms

352 conferred by the permeabilized brain methodology permit evaluation of lactate oxidation

353 in this tissue, and adds to the techniques with which mitochondrial function in brain can

354 be explored to investigate pathologies, test various applications, and describe the 355 phenotypic outcomes of transgenic animal models.

\section{Acknowledgments}

360 Thanks to Mary Weir and Devin Manning for technical assistance, and Dr. Anthony

361 Hickey (The University of Auckland) and Dr. L. Bruce Gladden (Auburn University) for

362 each commenting on earlier drafts of this work. Portions of this work were presented at

363 the 9th annual Mitochondrial Physiology School, Greenville, NC, August, 2015.

364

365 Declarations of interest

366 Authors declare no conflicts of interest. 


\section{Funding information}

368 This work was supported by grants from The Natural Sciences and Engineering Research

369 Council of Canada, The Nova Scotia Health Research Foundation, and The Canada

370 Foundation for Innovation.

371

372 Author contributions. All experiments involving mice were conducted in the laboratory

373 of GPH at the University of Guelph. Experiments involving rats were conducted in the

374 laboratories of DAK and $\mathrm{KB}$ at St. Francis Xavier University. EAFH and DAK

375 conceived the study; EAFH, MAJG, KB, GPH and DAK contributed to the design of the

376 study; EAFH, MAJG and DAK acquired, analyzed and interpreted the experimental data

377 for the study; EAFH, MAJG, and DAK drafted the manuscript; EAFH, MAJG, KB, GPH

378 and DAK revised the manuscript critically for intellectual content.

379

380

381

382

383

384

385

386

387

388

389 


\section{References}

391 Bittar, P.G., Charnay, Y., Pellerin, L., Bouras, C., and Magistretti, P.J. 1996. Selective

392 distribution of lactate dehydrogenase isoenzymes in neurons and astrocytes of human

393 brain. J. Cereb. Blood Flow Metab. 16(6): 1079-1089.

394 Blacker, T.S., and Duchen, M.R. 2016. Investigating mitochondrial redox state using

395 NADH and NADPH autofluorescence. Free Radic. Biol. Med. 100: 53-65.

396 Boumezbeur, F., Petersen, K.F., Cline, G.W., Mason, G.F., Behar, K.L., Shulman, G.I.,

397 and Rothman, D.L. 2010. The contribution of blood lactate to brain energy metabolism in

398 humans measured by dynamic ${ }^{13} \mathrm{C}$ nuclear magnetic resonance spectroscopy. J. Neurosci.

399 30(42): 13983-91.

400 Bouzier-Sore, A.-K., Voisin, P., Canioni, P., Magistretti, P.J., and Pellerin, L. 2003.

401 Lactate is a preferential oxidative energy substrate over glucose for neurons in culture. J.

402 Cereb. Blood Flow Metab. 23: 1298-1306.

403 Brooks, G.A. 1985. Lactate: glycolytic end product and oxidative substrate during

404 sustained exercise in mammals — the "lactate shuttle." In Circ. Respir. Metab. (pp 208-

405 218). Springer, Berlin, Heidelberg.

406 Brooks, G.A., Brown, M.A., Butz, C.E., Sicurello, J.P., and Dubouchaud, H. 1999a.

407 Cardiac and skeletal muscle mitochondria have a monocarboxylate transporter MCT1. J.

408 Appl. Physiol. 87(5): 1713-1718.

409 Brooks, G.A., Dubouchaud, H., Brown, M., Sicurello, J.P., and Butz, C.E. 1999b. Role of

410 mitochondrial lactate dehydrogenase and lactate oxidation in the intracellular lactate

411 shuttle. Proc. Natl. Acad. Sci. 96(3): 1129-1134. 
412 Cerdan, S., Rodrigues, T.B., Sierra, A., Benito, M., Fonseca, L.L., Fonseca, C.P., and

413 Garcia-Martin, M.L. 2006. The redox switch/redox coupling hypothesis. Neurochem. Int.

$414 \quad 48(6-7): 523-530$.

415 Chen, Y.-J., Mahieu, N.G., Huang, X., Singh, M., Crawford, P.A., Johnson, S.L., Gross,

416 R.W., Schaefer, J., and Patti, G.J. 2016. Lactate metabolism is associated with

417 mammalian mitochondria. Nat. Chem. Biol. 12(11): 937-943.

418 Devin, A., Guerin, B., and Rigoulet, M. 1997. Cytosolic NAD ${ }^{+}$content strictly depends

419 on ATP concentration in isolated liver cells. FEBS Lett. 410(2-3): 329-332.

420 Dienel, G. A. 2012. Brain lactate metabolism: the discoveries and the controversies. J.

421 Cereb. Blood Flow Metab. 32(7): 1107-1138.

422 Dubouchaud, H., Butterfield, G.E., Wolfel, E.E., Bergman, B.C., and Brooks, G. a. 2000.

423 Endurance training, expression, and physiology of LDH, MCT1, and MCT4 in human

424 skeletal muscle. Am. J. Physiol. Endocrinol. Metab. 278(4): 571-579.

425 Elustondo, P.A., White, A.E., Hughes, M.E., Brebner, K., Pavlov, E., and Kane, D.A.

426 2013. Physical and functional association of lactate dehydrogenase (LDH) with skeletal

427 muscle mitochondria. J. Biol. Chem. 288(35): 25309-17.

428 Gladden, L.B. 2004. Lactate metabolism: a new paradigm for the third millennium. J.

429 Physiol. 558(1): 5-30.

430 Gladden, L.B. 2007. Is there an intracellular lactate shuttle in skeletal muscle? J. Physiol.

$431 \quad$ 582(Pt 3): 899.

432 Gladden, L.B. 2008. A “lactatic” perspective on metabolism. Med. Sci. Sports Exerc.

$433 \quad 40(3), 477-485$. 
434 Gray, L.R., Tompkins, S.C., and Taylor, E.B. 2014. Regulation of pyruvate metabolism

435 and human disease. Cell. Mol. Life Sci. 71(14), 2577-2604.

436 Halestrap, A. 1975. The mitochondrial pyruvate carrier. Kinetics and specificity for

437 substrates and inhibitors. Biochem. J. 148(1): 85-96.

438 Halestrap, A.P., and Denton, R.M. 1974. Specific inhibition of pyruvate transport in rat

439 liver mitochondria and human erythrocytes by alpha-cyano-4-hydroxycinnamate.

440 Biochem. J. 138(2): 313-316.

441 Hashimoto, T., Hussien, R., and Brooks, G. A. 2006. Colocalization of MCT1, CD147,

442 and LDH in mitochondrial inner membrane of L6 muscle cells: evidence of a

443 mitochondrial lactate oxidation complex. Am. J. Physiol. Endocrinol. Metab. 290(6):

444 E1237-E1244.

445 Hashimoto, T., Hussien, R., Cho, H.S., Kaufer, D., and Brooks, G.A. 2008. Evidence for

446 the mitochondrial lactate oxidation complex in rat neurons: Demonstration of an essential

447 component of brain lactate shuttles. PLoS One, 3(8): e2915.

448 Herbst, E.A., and Holloway, G.P. 2016. Exercise increases mitochondrial glutamate

449 oxidation in the mouse cerebral cortex. Appl. Physiol. Nutr. Metab. 41(7): 799-801.

450 Herbst, E.A.F., and Holloway, G.P. 2015. Permeabilization of brain tissue in situ enables

451 multiregion analysis of mitochondrial function in a single mouse brain. J. Physiol.

$452 \quad$ 593(4): 787-801.

453 Hui, S., Ghergurovich, J., Morscher, R., Jang, C., Teng, X., Lu, W., Esparza, L., Reya,

454 T., Yanxiang, G., White, E., and Rabinowitz, J. 2017. Glucose feeds the TCA cycle via

455 circulating lactate. Nature, 551(7678): 115-118. 
456 Jacobs, R.A., Meinild, A.-K., Nordsborg, N.B., and Lundby, C. 2013. Lactate oxidation

457 in human skeletal muscle mitochondria. Am. J. Physiol. Endocrinol. Metab. 304(7):

458 E686-94.

459 Jones, D.P., and Sies, H. 2015. The Redox Code. Antioxid. Redox Signal. 23(9): 734-

460746.

461 Kane, D.A. 2014. Lactate oxidation at the mitochondria: A lactate-malate-aspartate

462 shuttle at work. Front. Neurosci. 8: 366.

463 Kiss, D.S., Toth, I., Jocsak, G., Sterczer, A., Bartha, T., Frenyo, L. V., and Zsarnovszky,

464 A. 2016. Preparation of purified perikaryal and synaptosomal mitochondrial fractions

465 from relatively small hypothalamic brain samples. MethodsX, (3): 417-429.

466 Kras, K.A., Willis, W.T., Barker, N., Czyzyk, T., Langlais, P.R., and Katsanos, C.S.

467 2016. Subsarcolemmal mitochondria isolated with the proteolytic enzyme nagarse exhibit

468 greater protein specific activities and functional coupling. Biochem. Biophys. Reports, 6:

469 101-107.

470 Kristian, T. 2010. Isolation of mitochondria from the CNS. Curr. Protoc. Neurosci.

$471 \quad 52: 7.22: 7.22 .1-7.22 .12$.

472 Di Lisa, F., and Ziegler, M. 2001. Pathophysiological relevance of mitochondria in NAD ${ }^{+}$

473 metabolism. FEBS Lett. 492(1-2), 4-8.

474 Martin-Requero, A., Ayuso, M.S., and Parilla, R. 1986. Rate-limiting steps for hepatic

475 gluconeogenesis. Mechanisms of oxamate inhibition of mitochondrial pyruvate

476 metabolism. J. Biol. Chem. 261(30): 13973-13978.

477 Passarella, S., de Bari, L., Valenti, D., Pizzuto, R., Paventi, G., and Atlante, A. 2008.

478 Mitochondria and 1-lactate metabolism. FEBS Lett. 582(25-26), 3569-3576. 
479 Passarella, S., Paventi, G., and Pizzuto, R. 2015. The mitochondrial L-lactate

480 dehydrogenase affair. Front. Neurosci. 8: 407.

481 Paventi, G., Pizzuto, R., and Passarella, S. 2017. The occurance of L-lactate

482 dehydrogenase in the inner mitochondrial compartment of pig liver. Biochem. Biophys.

483 Res. Commun. 489: 255-261.

484 Pellerin, L., and Magistretti, P.J. 2012. Sweet sixteen for ANLS. J. Cereb. Blood Flow

485 Metab. 32(7): 1152-1166.

486 Perry, C.G.R., Kane, D.A., Lanza, I.R., and Neufer, P.D. 2013. Methods for assessing

487 mitochondrial function in diabetes. Diabetes, 62(4), 1041-1053

488 Ponsot, E., Zoll, J., N’Guessan, B., Ribera, F., Lampert, E., Richard, R., Veksler, V.,

489 Ventura-Clapier, R., and Mettauer, B. 2005. Mitochondrial tissue specificity of substrates

490 utilization in rat cardiac and skeletal muscles. J. Cell. Physiol. 203(3): 479-486.

491 Poole, R.C., and Halestrap, A.P. 1993. Transport of lactate and other monocarboxylates

492 across mammalian plasma membranes. Am. J. Physiol. 264(4), C761-C782.

493 Rasmussen, H.N., van Hall, G., and Rasmussen, U.F. 2002. Lactate dehydrogenase is not

494 a mitochondrial enzyme in human and mouse vastus lateralis muscle. J. Physiol. 541(2):

$495 \quad 575-580$.

496 Rogatzki, M.J., Ferguson, B.S., Goodwin, M.L., and Gladden, L.B. 2015. Lactate is

497 always the end product of glycolysis. Front. Neurosci. 9: 22.

498 Sahlin, K., Fernström, M., Svensson, M., and Tonkonogi, M. 2002. No evidence of an

499 intracellular lactate shuttle in rat skeletal muscle. J. Physiol. 541(2), 569-574.

500 Schurr, A. 2017. Lactate, Not Pyruvate, Is the End Product of Glucose Metabolism via

501 Glycolysis. In Carbohydrate. InTech. Prof. Mahmut Caliskan (Ed.) 
502 Simpson, I., Carruthers, A., and Vannucci, S.J. 2007. Supply and demand in cerebral 503 energy metabolism: the role of nutrient transporters. J. Cereb. Blood. Flow. Metab.

504 27(11): 1766-1791.

505 Skilleter, D.N., and Kun, E. 1972. The oxidation of l-lactate by liver mitochondria. Arch. 506 Biochem. Biophys. 152(1): 92-104.

507 Sun, F., Dai, C., Xie, J., and Hu, X. 2012. Biochemical issues in estimation of cytosolic 508 free NAD/NADH ratio. PLoS One, 7(5): e34525.

509 Szczesna-Kaczmarek, A. 1990. 1-Lactate oxidation by skeletal muscle mitochondria. Int.

510 J. Biochem. 22(6): 617-620.

511 Szczesna-Kaczmarek, A., Litwinska, D., and Popinigis, J. 1984. Oxidation of NADH via

512 an "external" pathway in skeletal -muscle mitochondria and its possible role in the

513 repayment of lactacid oxygen debt. Int. J. Biochem. 16(12): 1231-1235.

514 Taylor, E.B. 2017. Functional properties of the mitochondrial carrier system. Trend Cell 515 Biol. 27(9):633-644.

516 Tischler, M.E., Friedrichs, D., Coll, K., and Williamson, J.R. 1977. Pyridine nucleotide

517 distributions and enzyme mass action ratios in hepatocytes from fed and starved rats.

518 Arch. Biochem. Biophys. 184(1): 222-236.

519 Vinogradov, A., Scarpa, A., and Chance, B. 1972. Calcium and pyridine nucleotide 520 interaction in mitochondrial membranes. Arch. Biochem. Biophys. 152(2): 646-654.

521 Williamson, D.H., Lund, P., and Krebs, H. A. 1967. The redox state of free nicotinamide522 adenine dinucleotide in the cytoplasm and mitochondria of rat liver. Biochem. J. 103(2): $523 \quad 514-527$. 
524 Yoshida, Y., Holloway, G.P., Ljubicic, V., Hatta, H., Spriet, L.L., Hood, D.A., and

525 Bonen, A. 2007. Negligible direct lactate oxidation in subsarcolemmal and

526 intermyofibrillar mitochondria obtained from red and white rat skeletal muscle. J.

527 Physiol. 582(Pt 3): 1317-1335.

528

529

530

531

532

533

534

535

536

537

538

539

540

541

542

543

544

545

546 


\section{Figure Captions}

548 FIGURE 1. Titration of substrates in saponin-permeabilized mouse brain cortex. A, $549 \mathrm{NAD}^{+}$was titrated $(0-2.5 \mathrm{mM})$ atop $100 \mathrm{mM}$ lactate, $4 \mathrm{mM}$ malate and $5 \mathrm{mM}$ ADP.

550 Apparent $K_{\mathrm{m}}$ for $\mathrm{NAD}^{+}: 1.3 \pm 0.5 \mathrm{mM}$. B, Lactate was titrated $(0-100 \mathrm{mM})$ atop $2 \mathrm{mM}$ $551 \mathrm{NAD}^{+}, 4 \mathrm{mM}$ malate and $5 \mathrm{mM}$ ADP. Apparent $K_{\mathrm{m}}$ for lactate: $11.2 \pm 3.5 \mathrm{mM}$. Results 552 represent mean $\pm \mathrm{SD} ; n=4$.

553

554

555 FIGURE 2. Mitochondrial lactate oxidation in saponin-permeabilized mouse cortex 556 requires extramatrix $\mathrm{NAD}^{+}$and is comparable to glutamate and pyruvate-supported $\mathrm{JO}_{2}$.

557 A, $80 \mathrm{mM}$ lactate (+Lactate); $2.5 \mathrm{mM} \mathrm{NAD}^{+}\left(+\mathrm{NAD}^{+}\right)$or both $\left(+\right.$Lactate $\left.+\mathrm{NAD}^{+}\right)$were 558 added to $5 \mathrm{mM}$ ADP $+4 \mathrm{mM}$ malate. Respiration with $\mathrm{ADP}+$ malate + lactate $+\mathrm{NAD}^{+}$ 559 was greater than any other condition $\left({ }^{* * *} P<0.001 ; n=3\right)$. B, Mitochondrial $J_{2}$ was 560 comparable among $10 \mathrm{mM}$ glutamate, $10 \mathrm{mM}$ pyruvate and $80 \mathrm{mM}$ lactate, added atop 5 $561 \mathrm{mM} \mathrm{ADP}+4 \mathrm{mM}$ malate $+2.5 \mathrm{mM} \mathrm{NAD}^{+}$. Data are mean $\pm \mathrm{SD}, n=4$.

564 FIGURE 3. Effects of inhibitors of lactate dehydrogenase and mitochondrial pyruvate 565 transport on lactate-supported respiration in permeabilized mouse cortex. A, Oxamate $566(\mathrm{Oxm})$ was titrated $(10,20$ and $40 \mathrm{mM})$ in the presence of $5 \mathrm{mM}$ ADP $+4 \mathrm{mM}$ malate + $5672 \mathrm{mM} \mathrm{NAD}^{+}$and either $80 \mathrm{mM}$ lactate or $10 \mathrm{mM}$ pyruvate $(n=3-4) . * P<0.05, * * P<$ $5680.01,{ }^{*} P<0.001$ lactate $+\mathrm{NAD}^{+}$versus control (no Oxm). ${ }^{\# \#} P<0.001$ versus pyruvate + $56920 \mathrm{mM}$ Oxm condition; ${ }^{\# \# \#} P<0.0001$ versus pyruvate $+40 \mathrm{mM}$ Oxm condition. B, $\alpha-$ 
570 cyano-4-hydroxycinnamic acid (CHC) was titrated $(5,25$, and $50 \mu \mathrm{M})$ in the presence of

$5715 \mathrm{mM}$ ADP $+4 \mathrm{mM}$ malate $+2 \mathrm{mM} \mathrm{NAD}^{+}$and either $80 \mathrm{mM}$ lactate, $10 \mathrm{mM}$ pyruvate or

$57210 \mathrm{mM}$ glutamate $(n=3-4) .{ }^{* * *} P<0.001$ versus control for both lactate $+\mathrm{NAD}^{+}$and

573 pyruvate. ${ }^{\# \#} P<0.001$ glutamate versus each lactate $+\mathrm{NAD}^{+}$and pyruvate. Data are 574 mean \pm SD.

575

576

577 FIGURE 4. Mitochondrial respiration supported by exogenous $\mathrm{NAD}^{+}$-dependent lactate

578 oxidation in permeabilized rat brain subregions relies on the pyruvate carrier. A. 579 Representative oxygraphic trace of experiment: sequential additions of ADP (5 mM), 580 malate $(4 \mathrm{mM})$, lactate $(40 \mathrm{mM}), \mathrm{NAD}^{+}(4 \mathrm{mM}), \mathrm{UK}-5099(10 \mu \mathrm{M})$, glutamate $(10 \mathrm{mM})$, 581 and aminooxyacetate $(1 \mathrm{mM})$ were made in saponin-permeabilized prefrontal cortex 582 (PFC) and dorsal hippocampus (DH). Addition of lactate and $\mathrm{NAD}^{+}$increased 583 respiration, while the subsequent addition of the mitochondrial pyruvate transport 584 inhibitor UK-5099 decreased respiration. Glutamate respiration was unaffected by UK585 5099, but inhibited by aminooxyacetate. Dashed line $P<0.05$; dotted line $P<0.0001$ 586 between connecting points. ${ }^{* *} P<0.01 ;{ }^{* * *} P<0.001 ;{ }^{* * * *} P<0.0001 \mathrm{PFC}$ versus DH. ${ }^{\#} P<$ 587 0.05. Data are mean $\pm \mathrm{SD} ; n=10-11$. 
A

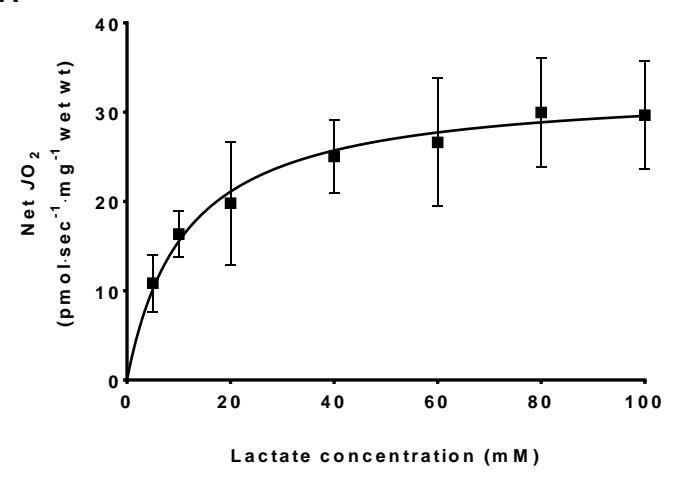

B

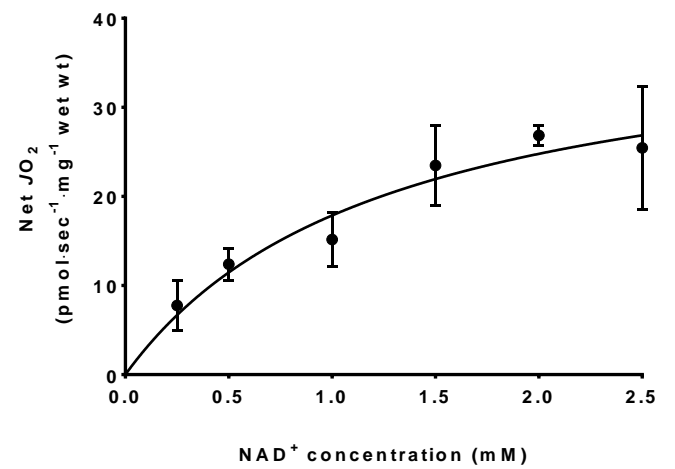


A

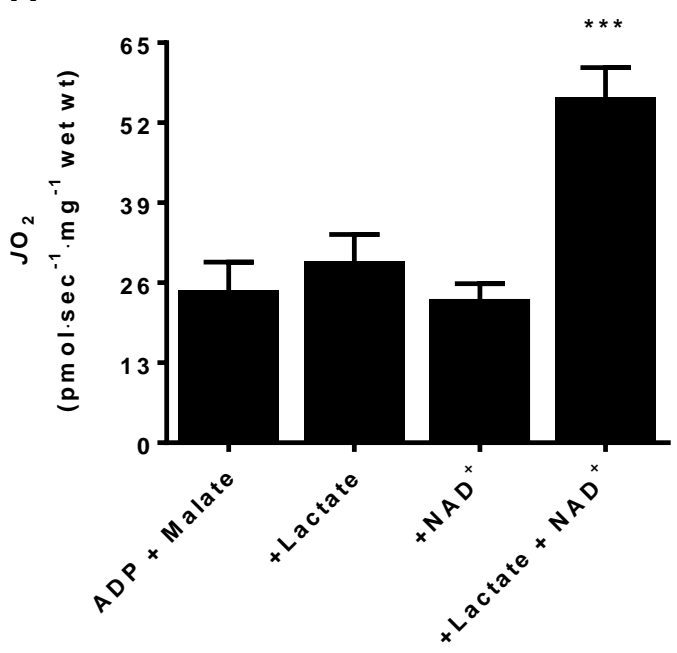

B

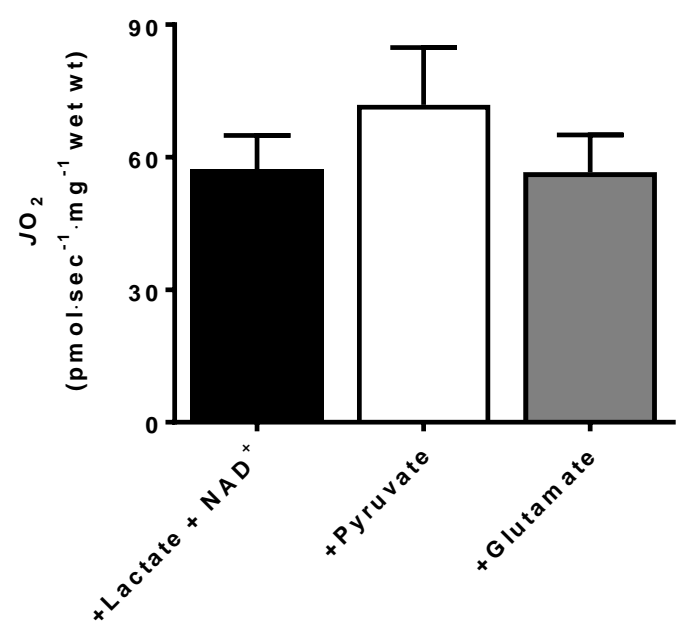



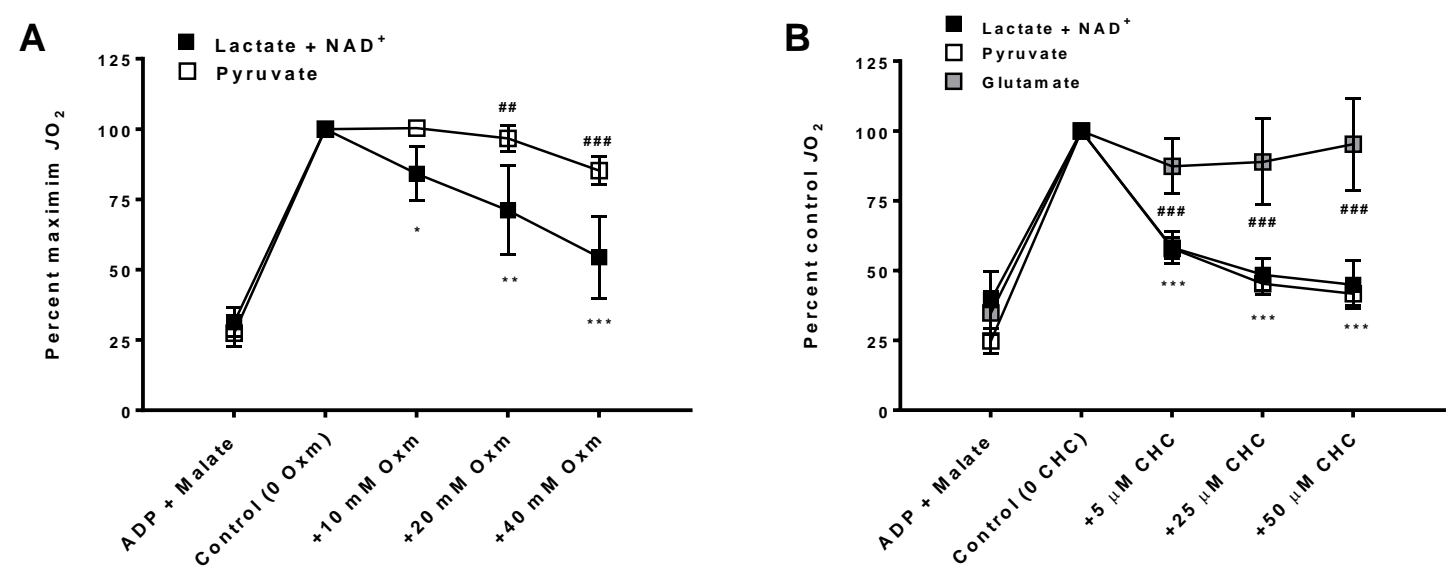
A.

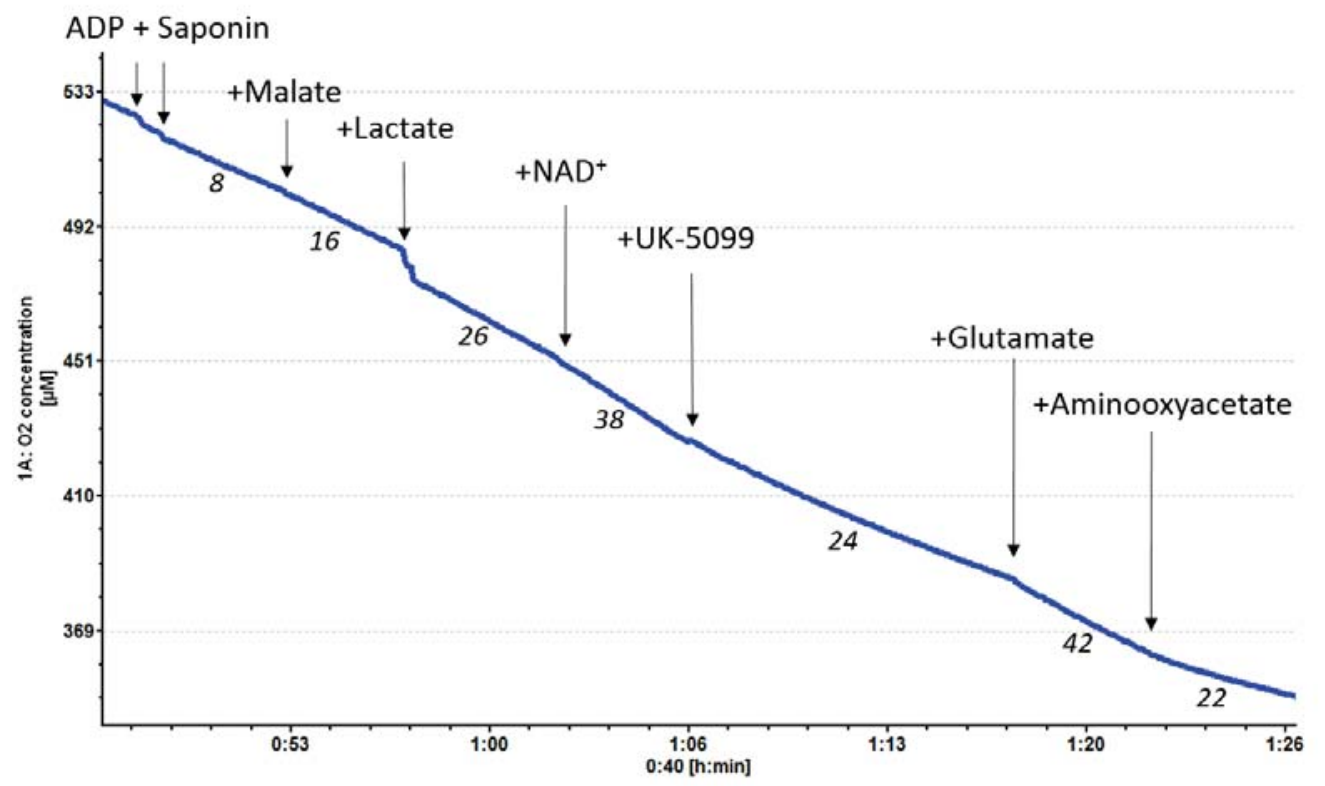

B.

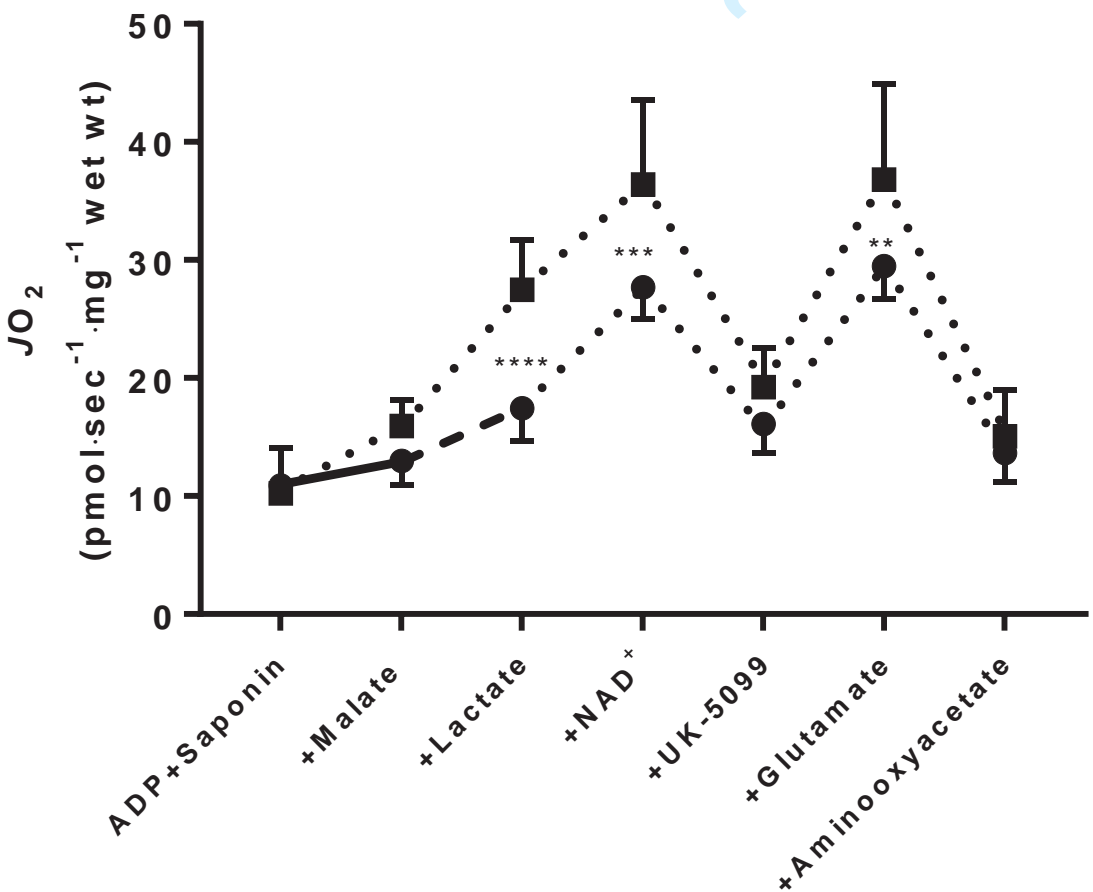

